\title{
EFFECTS OF NITRIC OXIDE SYNTHASE INHIBITOR (L-NAME) ON CYTOPATHOLOGIC CHANGES DUE TO CHOLESTASIS IN HEPATIC CELLS OF ADULT MALE RATS
}

\author{
AlireZA Monsef
}

Department of Pathology, Hamadan University of Medical Sciences, Hamadan, Iran

\begin{abstract}
Obstructive cholestasis is associated with overproduction of endogenous opioids, nitric oxide (NO) and cytokines in the blood stream. Nitro-L-arginine methyl ester (L-NAME) administration decreases the NO serum level and it is able to reduce related complications. The aim of this research is to survey the effects of the NO inhibitor on complications relating to cholestasis in liver cells and intrahepatic biliary ducts. We used five groups of animals: control, sham-operated (surgical control), bile duct ligated (BDL) group, BDL and normal saline infused group, and BDL with L-NAME administrated group. After 3 weeks all animals were killed, histopathology of liver cells and intrahepatic biliary ducts were evaluated by hematoxylin-eosin (HE), PAS (periodic acid-Schiff) and trichrome staining. The status of inflammation and fibrosis was evaluated by the modified Knodell score system.

Microscopic study of different groups showed that the necro-inflammatory score in the control group was 0.36 , it was 1 in the sham-operated group and it raised to 15.2 in the cholestatic group. After administration of L-NAME it had a meaningful decrease to 7, but in the saline-treated group, the score was 16. L-NAME with the mentioned dose was capable of decreasing the serum nitric oxide level, although it is able to decrease the unfavorable complications of cholestatic jaundice.
\end{abstract}

Key words: nitric oxide synthase, pathology, cholestasis, liver.

\section{Introduction}

Cholestasis is caused by obliteration of the common bile duct and ampulla of Vater due to bile stones, pancreatic head carcinoma, bile duct carcinoma or parasitic infestation. Cholestasis will cause some materials like bilirubin, bile acids and cholesterol to rise in blood. These materials normally secrete into bile. Recent investigations show that endogenous opioids, nitric oxide and cytokine levels also will rise in plasma. Clinical manifestations consisting of icterus, itching, fatigue, bone problems, respiratory function disturbances, peptic ulcer and renal failure may start as a consequence [1].

Brownish-green stippled appearance within the cytoplasm, representing bile stasis, is the microscopic view of individual hepatocytes involved. Canalicular bile plugs among hepatocytes or inside bile ducts may also be present, designating the extracted bile from the hepatocytes that is immobilized because of the obstruction. Tension caused by bile accumulation can cause the plugs to rupture, bile leaks into the surrounding tissue, and would cause hepatic necrosis. Bile canaliculi proliferations and portal space inflammation are also observed [1].

In 1995 , in a study by Niederberger, it was revealed that the normalization of nitric oxide production will cause regulation of arterial expansion in rats with cirrhosis [2]. The above-mentioned studies suggest that the whole or part of clinical problems and histopathologic changes are due to the increase in the nitric oxide (NO) serum level, so studies for evaluation of nitric oxide synthase inhibitors have been developed. In 
1999, El-Gohary et al. studied the effect of Voltametrin on apoptosis of rat testicular cells. Voltametrin caused serum NO to rise, in addition, the testicular cells showed apoptosis and inhibition of spermatogenesis. Nitric oxide synthase inhibitor drug (nitro-L-arginine methyl ester - L-NAME) at a dose of $1 \mathrm{mg} / \mathrm{kg}$ reduced these harmful effects [3].

In 2001, Mani et al. showed the effect of naloxone (opioid inhibitor) on the rats with cholestasis. It will protect the stomach from indometacin side effects [1]. Namiranian et al. displayed that nitric oxide synthase inhibitor (L-NAME) at a dose of $1 \mathrm{mg} / \mathrm{kg}$ did not have any effect on complications of cholestasis [4].

In this study we surveyed the effects of cholestasis on the liver and the effect of L-NAME as a nitric oxide synthase inhibitor on histopathologic changes of the mentioned cases.

\section{Material and methods}

The study is an experimental study. It was performed at the Histology Department of the Tehran University of Medical Sciences and Hamadan University of Medical Sciences in 2002 to 2003.

\section{Animals and treatment}

Male white Sprague-dawley healthy rats between 200 and 250 grams were used. All animals had free access to food and water. The animals were treated according to the criteria defined in the "Guide for the Care and Use of Laboratory Animals" (NIH US publication 86-23 revised 1985). 30 rats were randomly assigned to 5 experimental groups:

- I - control (untreated) group: control animals were kept under the same conditions as experimental groups, but treated without any drug;

- II - sham-operated group: rats with abdominal surgery but without bile duct ligation;

- III - Bile duct ligated (BDL) group: rats with abdominal surgery and bile duct ligation;

- IV - BDL and normal saline infused group: bile duct ligated rats infused with $1 \mathrm{mg} / \mathrm{kg}$ normal saline;

- V - BDL and L-NAME administered group: each rat was given $1 \mathrm{mg} / \mathrm{kg}$ of L-NAME daily for 21 days.

\section{Materials}

L-NAME as a nitric oxide synthase inhibitor was provided by Sigma-Aldrich (Sigma, St. Louis, MO, USA).

\section{Histological assessment of the grades of hepatitis and fibrosis}

Liver tissue sections were stained with HE, PAS and trichrome, then evaluated for the grades of inflammation and fibrosis. The status of inflammation and fibrosis was evaluated by the modified Knodell score system with the score ranging from 0 to 24 in this system. The status of inflammation was evaluated with a grading system of total $0-18$ :

- 1 - piecemeal necrosis (0-4),

- 2 - confluent necrosis (0-6),

- 3 - focal (spotty) lytic necrosis, apoptosis and focal inflammation (0-4),

- 4 - portal inflammation (0-4).

The status of fibrosis was evaluated with a staging system ranging 0-6:

- stage 0: no fibrosis,

- stage 1: fibrous expansion of some portal areas, with or without short fibrous septa,

- stage 2: fibrous expansion of most portal areas, with or without short fibrous septa,

- stage 3: fibrous expansion of most portal areas with occasional portal to portal bridging,

- stage 4: fibrous expansion of portal areas with marked portal-portal (p-p) as well as portal-central (p-c) bridging,

- stage 5: marked bridging ( $\mathrm{p}-\mathrm{p}$ and/or p-c) with occasional nodules (incomplete cirrhosis),

- stage 6: cirrhosis, probable or definite.

According to this scoring system of $\mathrm{z}$ modified histological activity index, grading: necroinflammatory scores range from 0 to 18 and for the staging of architectural changes, fibrosis, and cirrhosis, a range of scores of 0 to 6 is considered. $U$ test was used to analyze the scores achieved in each category between rat groups.

\section{Results}

The control group of rats' livers presents normal lobular pattern, portal triads and cellular elements. They had a score of 2 and their mean was 0.33 of 24 (Table I). The sham-operated group was also similar to the control group and there were no significant differences between them. Their cumulative score was 6 and their mean was 0.5. All of the 6 cases of the BDL group reveal distorted lobular architecture. There was severe mononuclear infiltration in the majority of cases in the portal and periportal area, piecemeal necrosis and apoptosis, but in some cases it was moderate. All of them revealed a few necrotic hepatocytes and mild to moderate fibrosis and edema also has been noted. There were apoptotic hepatocytes, whereas bile duct hyperplasia was not revealed. About half of the hepatocytes showed bile pigment and bile duct hyperplasia was seen in 2 out of 6 cases and the other 4 cases showed severe bile duct hyperplasia. There was no difference in binuclear hepatocytes in comparison to the control group. We found multinuclear hepatocytes in 2 cases. Their overall score was 91 and the mean was 15.2 of 24 (Table II).

The fourth group which received daily injections of normal saline after cholestasis, did not show signi- 
Table I. Necro-inflammatory scores in the control group of rats

\begin{tabular}{lllllll}
\hline CASE & 1 & 2 & 3 & 4 & 5 & 6 \\
\hline Piecemeal necrosis (0-4) & 0 & 0 & 0 & 0 & 0 & 0 \\
\hline Confluent necrosis (0-6) & 0 & 0 & 0 & 0 & 0 & 0 \\
\hline Focal lytic necrosis \& apoptosis (0-4) & 0 & 1 & 0 & 0 & 0 & 0 \\
\hline Portal inflammation (0-4) & 0 & 1 & 0 & 0 & 0 & 0 \\
\hline Architectural changes \& fibrosis (0-6) & 0 & 0 & 0 & 0 & 0 & 0 \\
\hline Sum & 0 & 2 & 0 & 0 & 0 & 0 \\
\hline Mean & $\mathbf{0 . 3 3}$ (SD = 0.81) & & &
\end{tabular}

Table II. Necro-inflammatory score in the cholestatic group

\begin{tabular}{lcccccc}
\hline CASE & 1 & 2 & 3 & 4 & 5 & 6 \\
\hline Piecemeal necrosis (0-4) & 4 & 4 & 3 & 4 & 4 & 3 \\
\hline Confluent necrosis (0-6) & 2 & 2 & 2 & 3 & 3 & 3 \\
\hline Focal lytic necrosis \& apoptosis (0-4) & 4 & 4 & 2 & 3 & 3 & 3 \\
\hline Portal inflammation (0-4) & 2 & 2 & 3 & 4 & 4 & 3 \\
\hline Architectural changes \& fibrosis (0-6) & 2 & 3 & 3 & 3 & 3 & 3 \\
\hline Sum & 14 & 15 & 13 & 17 & & 17 \\
\hline Mean & $15.16($ SD $=1.6)$ & & & &
\end{tabular}

Table III. Necro-inflammatory score in the L-NAME treated group

\begin{tabular}{lllllll}
\hline CASE & 1 & 2 & 3 & 4 & 5 & 6 \\
\hline Piecemeal necrosis (0-4) & 2 & 2 & 1 & 1 & 1 & 2 \\
\hline Confluent necrosis (0-6) & 2 & 2 & 1 & 1 & 1 & 1 \\
\hline Focal lytic necrosis \& apoptosis (0-4) & 2 & 2 & 1 & 1 & 1 & 1 \\
\hline Portal inflammation (0-4) & 2 & 2 & 1 & 1 & 1 & 2 \\
\hline Architectural changes \& fibrosis (0-6) & 1 & 2 & 1 & 5 & 5 & 7 \\
\hline Sum & 9 & 10 & 5 & & \\
\hline Mean & $6.83($ SD $=2.22)$ & & & &
\end{tabular}

ficant differences as compared to the cholestasis group. We found distorted lobular architecture, severe portal inflammation, necrosis, apoptosis and bile duct hyperplasia. Their cumulative score was 97 and mean score was 16 of 24 . The fifth group received daily L-NAME after cholestasis. The cholestasis had markedly diminished. The lobular architecture had been preserved in 5 out of 6 cases and in one of them it was relatively normal. The portal inflammation was mild in 4 out of 6 cases and 2 of them no inflammation was revealed. There was no necrosis and fibrosis in any section. Apoptosis was found at a rate of 3 to 4 cells in every lobule and bile duct epithelial cells intended for apoptosis. Bile duct hyperplasia was noted to a moderate to severe degree. The cumulative score of this group was 41 and mean score was 6.83 of 24 (Table III).

Portal inflammation and fibrosis in the cholestatic group was significantly higher than in the normal group using $\mathrm{U}$ test, $\mathrm{t}=0.0035$ and $\mathrm{t}=0.0025$, respectively (Figs. 1 and 2). Lobular inflammation and liver fibrosis were meaningfully higher in the cholestatic group as compared to the L-NAME treated group, $\mathrm{t}=0.0044$ and $\mathrm{t}=0.0063$, respectively. The necroinflammatory score changes of three rat groups are summarized in Fig. 3.

\section{Discussion}

Cholestasis induced in rats by ligation of the common bile duct was categorized into five groups for evaluation of nitric oxide synthase effects. We have used the modified Knodell score numeral system for grading and staging of liver injury as an alternative to terms usually used in hepatology such as chronic persistent hepatitis $(\mathrm{CPH})$, chronic active hepatitis $(\mathrm{CAH})$ and chronic lobular hepatitis (CLH), and cirrhosis to nu- 


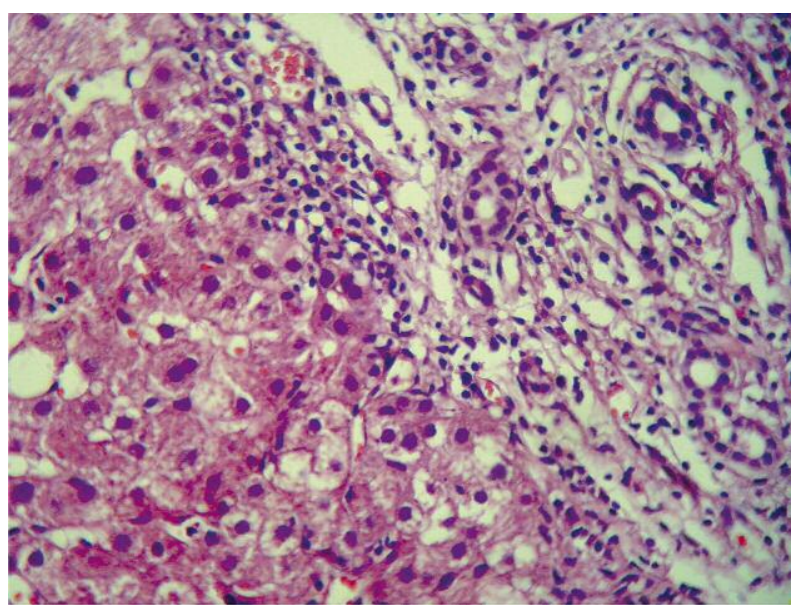

Fig. 1. Portal lymphocytic infiltration, destruction of the limiting plate and small bile duct proliferation in bile duct obstruction by ligation

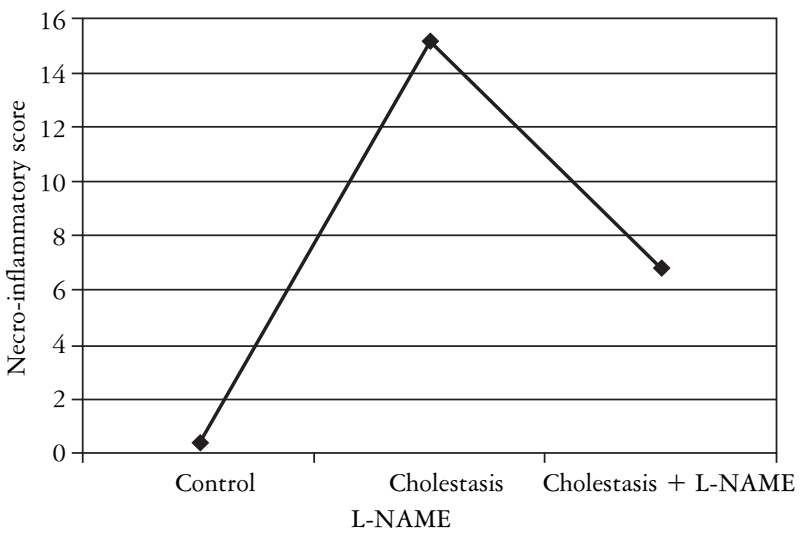

Fig. 3. Changes in necroinflammatory score in three groups of rats

merical system (0-24) and statistically analyzed them according to the method described elsewhere [5-7].

The data of previous studies suggested that cholestasis increases the levels of opioids, nitric oxide $(\mathrm{NO})$ and cytokines in serum. It also decreases the concentration of FSH, LH and the testosterone level [8]. Nitric oxide is harmful to Leydig cells. The study of Swain $e t$ al. did not reveal the effects of L-NAME in $1 \mathrm{mg} / \mathrm{kg}$ dosage on cholestasis complications [8]. Another study showed that oxidative stresses had markedly increased in cholestatic and diabetic patients, and it may be due to inhibition of anti-oxidative activity by hyperglycemia [9]. This study shows that cholestasis results in an architectural distortion of liver structure, intrahepatic bile duct proliferation, accumulation of bile pigments in hepatocytes and liver cell apoptosis. In our study, the cholestasis leads to a 9-point increase of the Knodell score in the BDL group comparing to the control group of rats; the difference was marked and meaningful. In fact, the cholestasis caused a status similar to $\mathrm{CAH}$ in the $\mathrm{BDL}$ rats' livers.

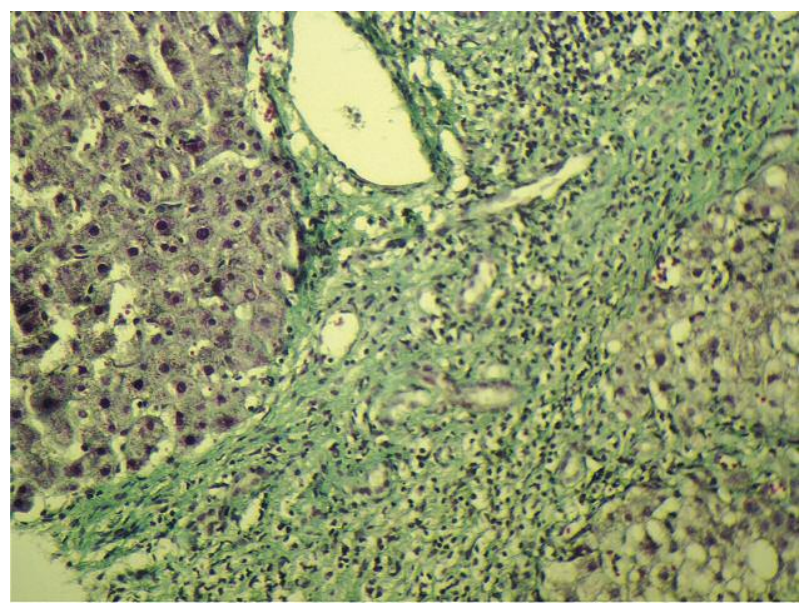

Fig. 2. The same case revealed fibrosis developed in the periportal area

Fibrosis and portal inflammation were higher in the cholestatic group than the normal group in our study. Apoptosis or programmed liver cell necrosis is not only dependent on cholestasis, but the increased apoptosis rate in the cholestatic rat group (in comparison to the normal group) suggests the effective role of cholestasis.

The results of our study showed that $1 \mathrm{mg} / \mathrm{kg}$ dosage of L-NAME decreased apoptosis by about $50 \%$ in the liver. The Knodell score was $0.36 / 24$ in the normal group, $1 / 24$ in sham-operated and 15.2/24 in the cholestatic group. In the L-NAME treated group, the score was $7 / 24$. The changes modified the pathologic status of rat liver from CAH in the BDL group to $\mathrm{CPH}$ in the L-NAME treated group. Lobular inflammation and fibrosis were meaningfully improved in the L-NAME treated group in comparison to the cholestatic group. These findings are similar to results reported by other investigators [10-12].

In 1999, El-Gohary et al. [3] demonstrated that administration of Deltametrine increases nitric oxide concentration in serum and leads to apoptosis in rat testis. The administration of L-NAME at $1 \mathrm{mg} / \mathrm{kg}$ dose decreased apoptosis [3]. Other studies indicated that administration of guanidine as a nitric oxide inhibitor reduces the rate of apoptosis [4, 13]. In this study, Namiranian $e t$ al. showed that L-NAME at $1 \mathrm{mg} / \mathrm{kg}$ dosage did not have meaningful effects on the testis of cholestatic rats.

Del Punta et al. [14] and Navarro [13] concluded that an increased level of nitric oxide in the process of obstructive jaundice leads to a reduction in cellular energy via impairment in the mitochondrial function.

Therefore, any agent that has the capacity of normalizing the nitric oxide level would be able to decrease the destructive process of cholestasis. Our study displayed the effectiveness of L-NAME in rat liver cells. It decreased cholestatic complications, portal inflam- 
mation and also promotes tissue structure whereas tissue necrosis is diminished. Our findings suggest that L-NAME with the mentioned dose is capable of decreasing the serum nitric oxide level, and thus it is able to decrease the unfavorable complications of cholestatic jaundice.

The author wishes to thank Mr. Moradi, Dr. Nasiri, Dr. Noori for their cooperation in the research process and the Hamadan University of Medical Sciences for the financial support. I also wish to thank the Farzan Institute for Research and Technology for technical assistance.

The author declares no conflict of interest.

\section{References}

1. Mani AR, Nahavandi A, Mani AH, Dehpour AR. Role of nitric oxide in hypodipsia in rats with obstructive cholestasis. J Phaarms Pharmacol 2001; 53: 277-281.

2. Niederberger M, Martin PY, Ginès P, et al. Normalization of nitric oxide production corrects arteria Vasoliation hyper dynamic circulation in cirrhotic rats. Gastroentrology 1995; 109: 1624-1630.

3. El-Gohary M, Awara WM, Nassar S, Hawas S. Deltametrin induced testicular apoptosis in rats, the protective effect of nitric oxide synthase inhibitors. Toxicology 1999; 132: 1-8.

4. Namiranian K, Samini M, Mehr SE, et al. Mesenteric vascular bed responsiveness in bile duct-ligated rats: roles of opioid and nitric oxide systems. Eur J Pharmacol 2001; 423: 185-193.

5. Ishak KG. Chronic hepatitis, Morphology and nomenclature. Mod Pathol 1994; 7: 690-713.

6. Knodell RG, Ishak KG, Black WC, et al. Formulation and application of a numerical scoring system for assessing histological activity in asymptomatic chronic active hepatitis. Hepatology 1981; 1: 431-435.

7. Kleiner DE. The pathology of drug-induced liver injury. Semin Liver Dis 2009; 29: 364-372.

8. Swain MG, Patchev V, Vergalla J, et al. Suppression of hypothalamic pituitary-adrenal axis responsiveness to stress in a rat model of acute cholestasis. J Clin Invest 1993; 91: 1903-1908.

9. Rosai and Ackerman's Surgical Pathology. 6th ed. 2002; 682.

10. Jones EA, Bergasa NV. The pruritus of cholestasis from bile acids to opiates agonists. Hepatology 1990; 11: 884-887.

11. Schweizer W, Duda P, Tanner S, et al. Experimental atrophy/hypertrophy complex (AHC) of the liver. J Hepatol 1995; 23: 71-78.

12. Yangs S, Bae L. Estrogen increase eNOS and NOX release in human coronary artery endothelium. J Cardiovascular Pharmacol 2000; 36: 242-247.

13. Navarro VJ. Herbal and dietary suplement hepatotoxicity. Semin Liver Dis 2009; 29: 373-382.

14. Del Punta K, Charreau EH, Pignataro OP. Nitric oxide inhibits leydig cell steroid genesis. Endocrinology 1996; 137: 5337-5343.

\section{Address for correspondence}

Alireza Monsef

P.O.Box: 13185-1678

Tehran, Iran

tel. +9821 66439463

fax +982166423304

e-mail: monsef_ar2001@yahoo.com 